\title{
Fixed point of $\alpha-\psi$-contractive type mappings in uniform spaces
}

\author{
Muhammad Usman Ali ${ }^{1}$, Tayyab Kamran ${ }^{2}$ and Erdal Karapınar ${ }^{3,4^{*}}$
}

"Correspondence:

erdalkarapinar@yahoo.com

${ }^{3}$ Department of Mathematics, Atilim University, Incek, Ankara 06836, Turkey

${ }^{4}$ Nonlinear Analysis and Applied

Mathematics Research Group

(NAAM), King Abdulaziz University,

Jeddah, Saudi Arabia

Full list of author information is

available at the end of the article

\begin{abstract}
In this paper, we shall investigate the existence and uniqueness of a fixed point of $\alpha$ - $\psi$-contractive mappings in the context of uniform spaces. We shall also prove some common fixed point theorems by introducing the notion of $\alpha$-admissible pairs. We shall construct some examples to support our novel results.
\end{abstract}

MSC: 46T99; 47H10; 54H25

Keywords: $\alpha$ - $\psi$-contractive; $\alpha$-admissible maps

\section{Introduction}

One of the interesting metric fixed point results was given by Samet et al. [1] by introducing the notions of $\alpha$-admissible and $\alpha-\psi$-contractive type mappings. They reported results via these new notions, and they extended and unified most of the related existing metric fixed point results in the literature. In particular, the authors [2] showed that fixed point results via cyclic contractions are consequences of their related results. Naturally, many authors have started to investigate the existence and uniqueness of a fixed point theorem via admissible mappings and variations of the concept of $\alpha-\psi$-contractive type mappings, for reference see [1-19]. The notion of cyclic contraction was introduced by Kirk et al. [20]. The main advantage of the cyclic contraction is that the given mapping does not need to be continuous. It has been appreciated by several authors; see e.g. [21-26] and related references therein.

In this paper, we shall consider the characterization of the notions of $\alpha$ - $\psi$-contractive and $\alpha$-admissible mappings in the context uniform spaces. Further, we shall prove some fixed point theorems by using these concepts. We shall also use $\alpha$-admissible pairs to investigate the existence and uniqueness of a common fixed point in the setting of uniform spaces. We shall also establish some examples to illustrate the main results.

For the sake of completeness, we shall recollect some basic definitions and fundamental results. Let $X$ be a nonempty set. A nonempty family, $\vartheta$, of subsets of $X \times X$ is called a uniform structure of $X$ it satisfies the following properties:

(i) if $G$ is in $\vartheta$, then $G$ contains the diagonal $\{(x, x) \mid x \in X\}$;

(ii) if $G$ is in $\vartheta$ and $H$ is a subset of $X \times X$ which contains $G$, then $H$ is in $\vartheta$;

(iii) if $G$ and $H$ are in $\vartheta$, then $G \cap H$ is in $\vartheta$;

(iv) if $G$ is in $\vartheta$, then there exists $H$ in $\vartheta$, such that, whenever $(x, y)$ and $(y, z)$ are in $H$, then $(x, z)$ is in $G$;

(v) if $G$ is in $\vartheta$, then $\{(y, x) \mid(x, y) \in G\}$ is also in $\vartheta$. 
The pair $(X, \vartheta)$ is called a uniform space and the element of $\vartheta$ is called entourage or neighborhood or surrounding. The pair $(X, \vartheta)$ is called a quasiuniform space (see e.g. $[27,28]$ ) if property (v) is omitted.

Let $\Delta=\{(x, x) \mid x \in X\}$ be the diagonal of a nonempty set $X$. For $V, W \in X \times X$, we shall use the following setting in the sequel:

$$
V \circ W=\{(x, y) \mid \text { there exist } z \in X:(x, z) \in W \text { and }(z, y) \in V\}
$$

and

$$
V^{-1}=\{(x, y) \mid(y, x) \in V\}
$$

For a subset $V \in \vartheta$, a pair of points $x$ and $y$ are said to be $V$-close if $(x, y) \in V$ and $(y, x) \in V$. Moreover, a sequence $\left\{x_{n}\right\}$ in $X$ is called a Cauchy sequence for $\vartheta$, if, for any $V \in \vartheta$, there exists $N \geq 1$ such that $x_{n}$ and $x_{m}$ are $V$-close for $n, m \geq N$. For $(X, \vartheta)$, there is a unique topology $\tau(\vartheta)$ on $X$ generated by $V(x)=\{y \in X \mid(x, y) \in V\}$ where $V \in \vartheta$.

A sequence $\left\{x_{n}\right\}$ in $X$ is convergent to $x$ for $\vartheta$, denoted by $\lim _{n \rightarrow \infty} x_{n}=x$, if, for any $V \in \vartheta$, there exists $n_{0} \in \mathbb{N}$ such that $x_{n} \in V(x)$ for every $n \geq n_{0}$. A uniform space $(X, \vartheta)$ is called Hausdorff if the intersection of all the $V \in \vartheta$ is equal to $\Delta$ of $X$, that is, if $(x, y) \in V$ for all $V \in \vartheta$ implies $x=y$. If $V=V^{-1}$ then we shall say that a subset $V \in \vartheta$ is symmetrical. Throughout the paper, we shall assume that each $V \in \vartheta$ is symmetrical. For more details, see e.g. [27, 29-32].

Now, we shall recall the notions of $A$-distance and $E$-distance.

Definition 1.1 $[29,30]$ Let $(X, \vartheta)$ be a uniform space. A function $p: X \times X \rightarrow[0, \infty)$ is said to be an $A$-distance if, for any $V \in \vartheta$, there exists $\delta>0$ such that if $p(z, x) \leq \delta$ and $p(z, y) \leq \delta$ for some $z \in X$, then $(x, y) \in V$.

Definition 1.2 $[29,30]$ Let $(X, \vartheta)$ be a uniform space. A function $p: X \times X \rightarrow[0, \infty)$ is said to be an $E$-distance if

(i) $p$ is an $A$-distance,

(ii) $p(x, y) \leq p(x, z)+p(z, y), \forall x, y, z \in X$.

Example 1.3 $[29,30]$ Let $(X, \vartheta)$ be a uniform space and let $d$ be a metric on $X$. It is evident that $\left(X, \vartheta_{d}\right)$ is a uniform space where $\vartheta_{d}$ is a set of all subsets of $X \times X$ containing a 'band' $U_{\epsilon}=\left\{(x, y) \in X^{2} \mid d(x, y)<\epsilon\right\}$ for some $\epsilon>0$. Moreover, if $\vartheta \subseteq \vartheta_{d}$, then $d$ is an $E$-distance on $(X, \vartheta)$.

Lemma 1.4 [29, 30] Let $(X, \vartheta)$ be a Hausdorff uniform space and $p$ be an A-distance on $X$. Let $\left\{x_{n}\right\}$ and $\left\{y_{n}\right\}$ be sequences in $X$ and $\left\{\alpha_{n}\right\},\left\{\beta_{n}\right\}$ be sequences in $[0, \infty)$ converging to 0 . Then, for $x, y, z \in X$, the following results hold:

(a) If $p\left(x_{n}, y\right) \leq \alpha_{n}$ and $p\left(x_{n}, z\right) \leq \beta_{n}$ for all $n \in \mathbb{N}$, then $y=z$. In particular, if $p(x, y)=0$ and $p(x, z)=0$, then $y=z$.

(b) If $p\left(x_{n}, y_{n}\right) \leq \alpha_{n}$ and $p\left(x_{n}, z\right) \leq \beta_{n}$ for all $n \in \mathbb{N}$, then $\left\{y_{n}\right\}$ converges to $z$.

(c) If $p\left(x_{n}, x_{m}\right) \leq \alpha_{n}$ for all $n, m \in \mathbb{N}$ with $m>n$, then $\left\{x_{n}\right\}$ is a Cauchy sequence in $(X, \vartheta)$.

Let $p$ be an $A$-distance. A sequence in a uniform space $(X, \vartheta)$ with an $A$-distance is said to be a $p$-Cauchy if, for every $\epsilon>0$, there exists $n_{0} \in \mathbb{N}$ such that $p\left(x_{n}, x_{m}\right)<\epsilon$ for all $n, m \geq n_{0}$. 
Definition 1.5 $[29,30]$ Let $(X, \vartheta)$ be a uniform space and $p$ be an $A$-distance on $X$.

(i) $X$ is $S$-complete if, for every $p$-Cauchy sequence $\left\{x_{n}\right\}$, there exists $x$ in $X$ with $\lim _{n \rightarrow \infty} p\left(x_{n}, x\right)=0$.

(ii) $X$ is $p$-Cauchy complete if, for every $p$-Cauchy sequence $\left\{x_{n}\right\}$, there exists $x$ in $X$ with $\lim _{n \rightarrow \infty} x_{n}=x$ with respect to $\tau(\vartheta)$.

(iii) $T: X \rightarrow X$ is $p$-continuous if $\lim _{n \rightarrow \infty} p\left(x_{n}, x\right)=0$ implies $\lim _{n \rightarrow \infty} p\left(T\left(x_{n}\right), T(x)\right)=0$.

Remark 1.6 Let $(X, \vartheta)$ be a Hausdorff uniform space which is $S$-complete. If a sequence $\left\{x_{n}\right\}$ be a $p$-Cauchy sequence, then we have $\lim _{n \rightarrow \infty} p\left(x_{n}, x\right)=0$. Regarding Lemma 1.4(b), we derive $\lim _{n \rightarrow \infty} x_{n}=x$ with respect to the topology $\tau(\vartheta)$, and hence $S$-completeness implies $p$-Cauchy completeness.

Definition 1.7 [20] Let $X$ be a nonempty set, $m$ a positive integer and $T: X \rightarrow X$ a mapping. $X=\bigcup_{i=1}^{m} A_{i}$ is said to be a cyclic representation of $X$ with respect to $T$ if

(i) $A_{i}, i=1,2, \ldots, m$ are nonempty sets;

(ii) $T\left(A_{1}\right) \subset A_{2}, \ldots, T\left(A_{m-1}\right) \subset A_{m}, T\left(A_{m}\right) \subset A_{1}$.

\section{Main results}

Let $\Psi$ be the family of functions $\psi:[0, \infty) \rightarrow[0, \infty)$ satisfying the following conditions:

$\left(\Psi_{1}\right) \quad \psi$ is nondecreasing;

$\left(\Psi_{2}\right) \sum_{n=1}^{+\infty} \psi^{n}(t)<\infty$ for all $t>0$, where $\psi^{n}$ is the $n$th iterate of $\psi$.

These functions are known in the literature as (c)-comparison functions. It is easily proved that if $\psi$ is a (c)-comparison function, then $\psi(t)<t$ for any $t>0$.

Definition 2.1 [1] Let $T: X \rightarrow X$ and $\alpha: X \times X \rightarrow[0, \infty)$. We shall say that $T$ is $\alpha$-admissible if, for all $x, y \in X$, we have

$$
\alpha(x, y) \geq 1 \quad \Rightarrow \quad \alpha(T x, T y) \geq 1 .
$$

We shall characterize the notion of $\alpha-\psi$-contractive mapping, introduced by Samet et al. [1], in the context of uniform space as follows.

Definition 2.2 Let $(X, \vartheta)$ be a uniform space such that $p$ is an $E$-distance on $X$ and $T$ : $X \rightarrow X$ be a given mapping. We shall say that $T$ is an $\alpha-\psi$-contractive mapping if there exist two functions $\alpha: X \times X \rightarrow[0, \infty)$ and $\psi \in \Psi$ such that

$$
\alpha(x, y) p(T x, T y) \leq \psi(p(x, y)), \quad \text { for all } x, y \in X
$$

Theorem 2.3 Let $(X, \vartheta)$ be a S-complete Hausdorff uniform space such that $p$ be an E-distance on $X$. Let $T: X \rightarrow X$ be an $\alpha$ - $\psi$-contractive mapping satisfying the following conditions:

(i) $T$ is $\alpha$-admissible;

(ii) there exists $x_{0} \in X$ such that $\alpha\left(x_{0}, T x_{0}\right) \geq 1$ and $\alpha\left(T x_{0}, x_{0}\right) \geq 1$;

(iii) $T$ is p-continuous.

Then $T$ has a fixed point $u \in X$. 
Proof By hypothesis (ii) of the theorem we have $x_{0} \in X$ such that $\alpha\left(x_{0}, T x_{0}\right) \geq 1$. Define the sequence $\left\{x_{n}\right\}$ in $X$ by $x_{n+1}=T x_{n}$ for all $n \in \mathbb{N} \cup\{0\}$. If $x_{n_{0}}=x_{n_{0}+1}$ for some $n_{0}$, then $u=x_{n_{0}}$ is a fixed point of $T$. So, we can assume that $x_{n} \neq x_{n+1}$ for all $n$. Since $T$ is $\alpha$-admissible, we have

$$
\alpha\left(x_{0}, x_{1}\right)=\alpha\left(x_{0}, T x_{0}\right) \geq 1 \quad \Rightarrow \quad \alpha\left(T x_{0}, T x_{1}\right)=\alpha\left(x_{1}, x_{2}\right) \geq 1 .
$$

Inductively, we have

$$
\alpha\left(x_{n}, x_{n+1}\right) \geq 1, \quad \text { for all } n \in \mathbb{N} \cup\{0\} .
$$

From (2.1) and (2.2), it follows that, for all $n \in \mathbb{N}$, we have

$$
p\left(x_{n+1}, x_{n}\right)=p\left(T x_{n}, T x_{n-1}\right) \leq \alpha\left(x_{n}, x_{n-1}\right) p\left(T x_{n}, T x_{n-1}\right) \leq \psi\left(p\left(x_{n}, x_{n-1}\right)\right) .
$$

Iteratively, we derive

$$
p\left(x_{n}, x_{n+1}\right) \leq \psi^{n}\left(p\left(x_{0}, x_{1}\right)\right), \quad \text { for all } n \in \mathbb{N} \text {. }
$$

Since $p$ is an $E$-distance, for $m>n$, we have

$$
\begin{aligned}
p\left(x_{n}, x_{m}\right) & \leq p\left(x_{n}, x_{n+1}\right)+\cdots+p\left(x_{m-1}, x_{m}\right) \\
& \leq \psi^{n}\left(p\left(x_{0}, x_{1}\right)\right)+\psi^{n+1}\left(p\left(x_{0}, x_{1}\right)\right)+\cdots+\psi^{m-1}\left(p\left(x_{0}, x_{1}\right)\right) .
\end{aligned}
$$

To show that $\left\{x_{n}\right\}$ is a $p$-Cauchy sequence, consider

$$
S_{n}=\sum_{k=0}^{n} \psi^{k}\left(p\left(x_{0}, x_{1}\right)\right)
$$

Thus from (2.4) we have

$$
p\left(x_{n}, x_{m}\right) \leq S_{m-1}-S_{n-1} .
$$

Since $\psi \in \Psi$, there exists $S \in[0, \infty)$ such that $\lim _{n \rightarrow \infty} S_{n}=S$. Thus by (2.5) we have

$$
\lim _{n, m \rightarrow \infty} p\left(x_{n}, x_{m}\right)=0
$$

Since $p$ is not symmetrical, by repeating the same argument we have

$$
\lim _{n, m \rightarrow \infty} p\left(x_{m}, x_{n}\right)=0 .
$$

Hence the sequence $\left\{x_{n}\right\}$ is a $p$-Cauchy in the $S$-complete space $X$. Thus, there exists $u \in$ $X$ such that $\lim _{n \rightarrow \infty} p\left(x_{n}, u\right)=0$, which implies $\lim _{n \rightarrow \infty} x_{n}=u$. Since $T$ is $p$-continuous, we have $\lim _{n \rightarrow \infty} p\left(T x_{n}, T u\right)=0$, which implies that $\lim _{n \rightarrow \infty}\left(x_{n+1}, T u\right)=0$. Hence we have $\lim _{n \rightarrow \infty} p\left(x_{n}, u\right)=0$ and $\lim _{n \rightarrow \infty}\left(x_{n}, T u\right)=0$. Thus by Lemma 1.4(a) we have $u=T u$.

In the following theorem, we omit the $p$-continuity by replacing a suitable condition on the obtained iterative sequence. 
Theorem 2.4 Let $(X, \vartheta)$ be a S-complete Hausdorff uniform space such that $p$ is an E-distance on $X$. Let $T: X \rightarrow X$ be an $\alpha$ - $\psi$-contractive mapping satisfying the following conditions:

(i) $T$ is $\alpha$-admissible;

(ii) there exists $x_{0} \in X$ such that $\alpha\left(x_{0}, T x_{0}\right) \geq 1$ and $\alpha\left(T x_{0}, x_{0}\right) \geq 1$;

(iii) for any sequence $\left\{x_{n}\right\}$ in $X$ with $x_{n} \rightarrow x$ as $n \rightarrow \infty$ and $\alpha\left(x_{n}, x_{n+1}\right) \geq 1$ for each $n \in \mathbb{N} \cup\{0\}$, then $\alpha\left(x_{n}, x\right) \geq 1$ for each $n \in \mathbb{N} \cup\{0\}$.

Then $T$ has a fixed point $u \in X$.

Proof By following the proof of Theorem 2.3, we know that $\left\{x_{n}\right\}$ is a $p$-Cauchy in the $S$-complete space $X$. Thus, there exists $u \in X$ such that $\lim _{n \rightarrow \infty} p\left(x_{n}, u\right)=0$, which implies $\lim _{n \rightarrow \infty} x_{n}=u$. By using (2.1) and assumption (iii), we get

$$
\begin{aligned}
p\left(x_{n}, T u\right) & \leq p\left(x_{n}, x_{n+1}\right)+p\left(x_{n+1}, T u\right) \\
& \leq p\left(x_{n}, x_{n+1}\right)+\alpha\left(x_{n}, u\right) p\left(T x_{n}, T u\right) \\
& \leq p\left(x_{n}, x_{n+1}\right)+\psi\left(p\left(x_{n}, u\right)\right) .
\end{aligned}
$$

Letting $n \rightarrow \infty$ in above inequality, we shall have $\lim _{n \rightarrow \infty} p\left(x_{n}, T u\right)=0$. Hence we have $\lim _{n \rightarrow \infty} p\left(x_{n}, u\right)=0$ and $\lim _{n \rightarrow \infty} p\left(x_{n}, T u\right)=0$. Thus by Lemma 1.4(a) we have $u=T u$.

Example 2.5 Let $X=\left\{\frac{1}{n}: n \in \mathbb{N}\right\} \cup\{0\}$ be endowed with the usual metric $d$. Define $\vartheta=$ $\left\{U_{\epsilon} \mid \epsilon>0\right\}$. It is easy to see that $(X, \vartheta)$ is a uniform space. Define $T: X \rightarrow X$ by

$$
T x= \begin{cases}0 & \text { if } x=0, \\ \frac{1}{3 n+1} & \text { if } x=\frac{1}{n}: n>1, \\ 1 & \text { if } x=1,\end{cases}
$$

and $\alpha: X \times X \rightarrow[0, \infty)$ by

$$
\alpha(x, y)= \begin{cases}1 & \text { if } x, y \in X-\{1\} \\ 0 & \text { otherwise }\end{cases}
$$

and $\psi(t)=\frac{t}{3}$ for all $t \geq 0$. One can easily see that $T$ is $\alpha-\psi$-contractive and $\alpha$-admissible mapping. Also for $x_{0}=\frac{1}{2}$ we have $\alpha\left(x_{0}, T x_{0}\right)=\alpha\left(T x_{0}, x_{0}\right)=1$. Moreover, for any sequence $\left\{x_{n}\right\}$ in $X$ with $x_{n} \rightarrow x$ as $n \rightarrow \infty$ and $\alpha\left(x_{n-1}, x_{n}\right)=1$ for each $n \in \mathbb{N}$ we have $\alpha\left(x_{n}, x\right)=1$ for each $n \in \mathbb{N}$. Therefore by Theorem $2.4, T$ has a fixed point.

In the sequel, we shall investigate the uniqueness of a fixed point. For this purpose, we shall introduce the following condition.

(H) For all $x, y \in \operatorname{Fix}(T)$, there exists $z \in X$ such that $\alpha(z, x) \geq 1$ and $\alpha(z, y) \geq 1$.

Here, Fix $(T)$ denotes the set of fixed points of $T$.

The following theorem guarantees the uniqueness of a fixed point.

Theorem 2.6 Adding the condition $(\mathrm{H})$ to the hypothesis of Theorem 2.3 (respectively, Theorem 2.4), we obtain the uniqueness of fixed point of $T$. 
Proof Suppose, on the contrary, that $v \in X$ is another fixed point of $T$. From (H), there exists $z \in X$ such that

$$
\alpha(z, u) \geq 1 \quad \text { and } \quad \alpha(z, v) \geq 1 .
$$

Owing to the fact that $T$ is $\alpha$-admissible, from (2.10), we have

$$
\alpha\left(T^{n} z, u\right) \geq 1 \quad \text { and } \quad \alpha\left(T^{n} z, v\right) \geq 1, \quad \text { for all } n \in \mathbb{N} \cup\{0\} .
$$

We define the sequence $\left\{z_{n}\right\}$ in $X$ by $z_{n+1}=T z_{n}=T^{n} z_{0}$ for all $n \in \mathbb{N} \cup\{0\}$ and $z_{0}=z$. From (2.11) and (2.1), we have

$$
p\left(z_{n+1}, u\right)=p\left(T z_{n}, T u\right) \leq \alpha\left(z_{n}, u\right) p\left(T z_{n}, T u\right) \leq \psi\left(p\left(z_{n}, u\right)\right),
$$

for all $n \in \mathbb{N} \cup\{0\}$. This implies that

$$
p\left(z_{n}, u\right) \leq \psi^{n}\left(p\left(z_{0}, u\right)\right), \quad \text { for all } n \in \mathbb{N} \text {. }
$$

Letting $n \rightarrow \infty$ in the above inequality, we obtain

$$
\lim _{n \rightarrow \infty} p\left(z_{n}, u\right)=0
$$

Similarly,

$$
\lim _{n \rightarrow \infty} p\left(z_{n}, v\right)=0
$$

From (2.13) and (2.14) together with Lemma 1.4(a), it follows that $u=v$. Thus we have proved that $u$ is the unique fixed point of $T$.

Definition 2.7 [9] A pair of two self-mappings $T, S: X \rightarrow X$ is said to be $\alpha$-admissible, if, for any $x, y \in X$ with $\alpha(x, y) \geq 1$, we have $\alpha(T x, S y) \geq 1$ and $\alpha(S x, T y) \geq 1$.

Definition 2.8 Let $(X, \vartheta)$ be a uniform space. A pair of two self-mappings $T, S: X \rightarrow X$ is said to be an $\alpha-\psi$-contractive pair if

$$
\alpha(x, y) \max \{p(T x, S y), p(S x, T y)\} \leq \psi(p(x, y)),
$$

for any $x, y \in X$, where $\psi \in \Psi$.

Theorem 2.9 Let $(X, \vartheta)$ be a S-complete Hausdorff uniform space such that $p$ is an

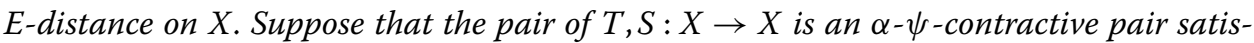
fying the following conditions:

(i) $(T, S)$ is $\alpha$-admissible;

(ii) there exists $x_{0} \in X$ such that $\alpha\left(x_{0}, T x_{0}\right) \geq 1$ and $\alpha\left(T x_{0}, x_{0}\right) \geq 1$;

(iii) for any sequence $\left\{x_{n}\right\}$ in $X$ with $x_{n} \rightarrow x$ as $n \rightarrow \infty$ and $\alpha\left(x_{n}, x_{n+1}\right) \geq 1$ for each $n \in \mathbb{N} \cup\{0\}$, then $\alpha\left(x_{n}, x\right) \geq 1$ for each $n \in \mathbb{N} \cup\{0\}$.

Then $T$ and $S$ have a common fixed point. 
Proof By hypothesis (ii) of the theorem, we have $x_{0} \in X$ such that $\alpha\left(x_{0}, T x_{0}\right) \geq 1$ and $\alpha\left(T x_{0}, x_{0}\right) \geq 1$. Since $(T, S)$ is an $\alpha$-admissible pair, we can construct a sequence such that

$$
\begin{aligned}
& T x_{2 n}=x_{2 n+1}, \quad S x_{2 n+1}=x_{2 n+2} \quad \text { and } \\
& \alpha\left(x_{n}, x_{n+1}\right) \geq 1, \quad \alpha\left(x_{n+1}, x_{n}\right) \geq 1, \quad \text { for all } n \in \mathbb{N} \cup\{0\} .
\end{aligned}
$$

From (2.15) for all $n \in \mathbb{N} \cup\{0\}$, we have

$$
\begin{aligned}
p\left(x_{2 n+1}, x_{2 n+2}\right) & =p\left(T x_{2 n}, S x_{2 n+1}\right) \\
& \leq \alpha\left(x_{2 n}, x_{2 n+1}\right) \max \left\{p\left(T x_{2 n}, S x_{2 n+1}\right), p\left(S x_{2 n}, T x_{2 n+1}\right)\right\} \\
& \leq \psi\left(p\left(x_{2 n}, x_{2 n+1}\right)\right) .
\end{aligned}
$$

Hence, we conclude that

$$
p\left(x_{2 n+1}, x_{2 n+2}\right) \leq \psi\left(p\left(x_{2 n}, x_{2 n+1}\right)\right) .
$$

Similarly, we find that

$$
\begin{aligned}
p\left(x_{2 n+2}, x_{2 n+3}\right) & =p\left(S x_{2 n+1}, T x_{2 n+2}\right) \\
& \leq \alpha\left(x_{2 n+1}, x_{2 n+2}\right) \max \left\{p\left(T x_{2 n+1}, S x_{2 n+2}\right), p\left(S x_{2 n+1}, T x_{2 n+2}\right)\right\} \\
& \leq \psi\left(p\left(x_{2 n+1}, x_{2 n+2}\right)\right) .
\end{aligned}
$$

Hence, we derive

$$
p\left(x_{2 n+2}, x_{2 n+3}\right) \leq \psi\left(p\left(x_{2 n+1}, x_{2 n+2}\right)\right) .
$$

Thus from (2.16) and (2.17), and by induction, we get

$$
p\left(x_{n}, x_{n+1}\right) \leq \psi^{n}\left(p\left(x_{0}, x_{1}\right)\right), \quad \text { for all } n \in \mathbb{N} \text {. }
$$

We shall show that $\left\{x_{n}\right\}$ is a $p$-Cauchy sequence, Since $p$ is an $E$-distance, for $m>n$, we have

$$
\begin{aligned}
p\left(x_{n}, x_{m}\right) & \leq p\left(x_{n}, x_{n+1}\right)+\cdots+p\left(x_{m-1}, x_{m}\right) \\
& \leq \psi^{n}\left(p\left(x_{0}, x_{1}\right)\right)+\psi^{n+1}\left(p\left(x_{0}, x_{1}\right)\right)+\cdots+\psi^{m-1}\left(p\left(x_{0}, x_{1}\right)\right) .
\end{aligned}
$$

Now, we shall consider

$$
S_{n}=\sum_{k=0}^{n} \psi^{k}\left(p\left(x_{0}, x_{1}\right)\right)
$$

Thus, from (2.19) we have

$$
p\left(x_{n}, x_{m}\right) \leq S_{m-1}-S_{n-1} .
$$


Since $\psi \in \Psi$, there exists $S \in[0, \infty)$ such that $\lim _{n \rightarrow \infty} S_{n}=S$. Thus, by (2.20) we have

$$
\lim _{n, m \rightarrow \infty} p\left(x_{n}, x_{m}\right)=0
$$

Since $p$ is not symmetrical, by repeating the same argument we have

$$
\lim _{n, m \rightarrow \infty} p\left(x_{m}, x_{n}\right)=0
$$

Hence the sequence $\left\{x_{n}\right\}$ is $p$-Cauchy in the $S$-complete space $X$. Thus, there exists $u \in X$ such that $\lim _{n \rightarrow \infty} p\left(x_{n}, u\right)=0$, which implies $\lim _{n \rightarrow \infty} T x_{2 n}=\lim _{n \rightarrow \infty} S x_{2 n+1}=u$. By using (2.15) and assumption (iii), we get

$$
\begin{aligned}
p\left(x_{n}, T u\right) & \leq p\left(x_{n}, x_{2 n+2}\right)+p\left(x_{2 n+2}, T u\right) \\
& =p\left(x_{n}, x_{2 n+2}\right)+p\left(S x_{2 n+1}, T u\right) \\
& \leq p\left(x_{n}, x_{2 n+2}\right)+\alpha\left(x_{2 n+1}, u\right) \max \left\{p\left(T x_{2 n+1}, S u\right), p\left(S x_{2 n+1}, T u\right)\right\} \\
& \leq p\left(x_{n}, x_{2 n+2}\right)+\psi\left(p\left(x_{2 n+1}, u\right)\right) .
\end{aligned}
$$

Letting $n \rightarrow \infty$ in (2.23), we have $p\left(x_{n}, T u\right)=0$. Hence we have $\lim _{n \rightarrow \infty} p\left(x_{n}, u\right)=0$ and $\lim _{n \rightarrow \infty} p\left(x_{n}, T u\right)=0$. Thus by Lemma 1.4(a) we have $u=T u$. Analogously, one can derive $u=S u$. Therefore $u=T u=S u$.

Remark 2.10 Note that Theorem 2.9 is valid if one replaces condition (ii) with

(ii) $)^{\prime}$ there exists $x_{0} \in X$ such that $\alpha\left(x_{0}, S x_{0}\right) \geq 1$ and $\alpha\left(S x_{0}, x_{0}\right) \geq 1$.

We shall get the following result by letting $S=T$ in Theorem 2.9.

Corollary 2.11 Let $(X, \vartheta)$ be a S-complete Hausdorff uniform space such that $p$ is an E-distance on $X$. Suppose that a mapping $T: X \rightarrow X$ is satisfying the condition

$$
\alpha(x, y) \max \{p(T x, y), p(x, T y)\} \leq \psi(p(x, y))
$$

for any $x, y \in X$, where $\psi \in \Psi$. Also suppose that the following conditions are satisfied:

(i) $T$ is $\alpha$-admissible;

(ii) there exists $x_{0} \in X$ such that $\alpha\left(x_{0}, T x_{0}\right) \geq 1$ and $\alpha\left(T x_{0}, x_{0}\right) \geq 1$;

(iii) for any sequence $\left\{x_{n}\right\}$ in $X$ with $x_{n} \rightarrow x$ as $n \rightarrow \infty$ and $\alpha\left(x_{n}, x_{n+1}\right) \geq 1$ for each $n \in \mathbb{N} \cup\{0\}$, then $\alpha\left(x_{n}, x\right) \geq 1$ for each $n \in \mathbb{N} \cup\{0\}$.

Then $T$ has a fixed point.

Example 2.12 Let $(X, d)$ is a dislocated metric space where $X=\left\{\frac{1}{n}: n \in \mathbb{N}\right\} \cup\{0\}$ and $d(x, y)=\max \{x, y\}$. Define $\vartheta=\left\{U_{\epsilon} \mid \epsilon>0\right\}$, where $U_{\epsilon}=\left\{(x, y) \in X^{2}: d(x, y)<d(x, x)+\epsilon\right\}$. It is easy to see that $(X, \vartheta)$ is a uniform space. Define $T: X \rightarrow X$ by

$$
T x= \begin{cases}0 & \text { if } x=0 \\ \frac{1}{2 n+1} & \text { if } x=\frac{1}{n}: n>1 \\ 1 & \text { if } x=1\end{cases}
$$


and $S: X \rightarrow X$ by

$$
S x= \begin{cases}0 & \text { if } x=0, \\ \frac{1}{2 n} & \text { if } x=\frac{1}{n}: n>1, \\ 1 & \text { if } x=1,\end{cases}
$$

and $\alpha: X \times X \rightarrow[0, \infty)$ by

$$
\alpha(x, y)= \begin{cases}1 & \text { if } x, y \in X-\{1\} \\ 0 & \text { otherwise }\end{cases}
$$

and $\psi(t)=\frac{t}{2}$ for all $t \geq 0$. One can easily see that $(T, S)$ is an $\alpha-\psi$-contractive and $\alpha$-admissible pair. Also for $x_{0}=\frac{1}{2}$ we have $\alpha\left(x_{0}, T x_{0}\right)=\alpha\left(T x_{0}, x_{0}\right)=1$. Moreover, for any sequence $\left\{x_{n}\right\}$ in $X$ with $x_{n} \rightarrow x$ as $n \rightarrow \infty$ and $\alpha\left(x_{n}, x_{n+1}\right) \geq 1$ for each $n \in \mathbb{N} \cup\{0\}$, we have $\alpha\left(x_{n}, x\right) \geq 1$ for each $n \in \mathbb{N}$. Therefore by Theorem 2.9, $T$ and $S$ have a common fixed point.

To investigate the uniqueness of a common fixed point, we shall introduce the following condition.

(I) For each $x, y \in \operatorname{CFix}(T, S)$, we have $\alpha(x, y) \geq 1$, where $\operatorname{CFix}(T, S)$ is the set of all common fixed points of $T$ and $S$.

Theorem 2.13 Adding the condition (I) to the hypothesis of Theorem 2.9, we obtain the uniqueness of the common fixed point of $T$ and $S$.

Proof On the contrary suppose that $u, v \in X$ are two distinct common fixed points of $T$ and $S$. From (I) and (2.15) we have

$$
p(u, v) \leq \alpha(u, v) \max \{p(T u, S v), p(S u, T v)\} \leq \psi(p(u, v))<p(u, v),
$$

which is impossible for $p(u, v)>0$. Consequently, we have $p(u, v)=0$. Analogously, one can show that $p(u, u)=0$. Thus we have $u=v$, which is a contradiction to our assumption. Hence $T$ and $S$ have a unique common fixed point.

\section{Consequences}

\subsection{Standard contractions on uniform space}

Taking in Theorem 2.6, $\alpha(x, y)=1$ for all $x, y \in X$, we shall obtain immediately the following fixed point theorems.

Corollary 3.1 Let $(X, \vartheta)$ be a S-complete Hausdorff uniform space such that $p$ is an E-distance on $X$ and $T: X \rightarrow X$ be a given mapping. Suppose that there exists a function $\psi \in \Psi$ such that

$$
p(T x, T y) \leq \psi(p(x, y))
$$

for all $x, y \in X$. Then $T$ has a unique fixed point. 
By substituting $\psi(t)=k t$, where $k \in[0,1)$, in Corollary 3.1, we shall get the following.

Corollary 3.2 Let $(X, \vartheta)$ be a S-complete Hausdorff uniform space such that $p$ is an E-distance on $X$. Suppose that $T: X \rightarrow X$ is a given mapping satisfying

$$
p(T x, T y) \leq k p(x, y)
$$

for all $x, y \in X$. Then $T$ has a unique fixed point.

Taking in Theorem $2.13 \alpha(x, y)=1$ for all $x, y \in X$, we shall obtain immediately the following common fixed point theorem.

Corollary 3.3 Let $(X, \vartheta)$ be a S-complete Hausdorff uniform space such that $p$ is an E-distance on $X$ and $T, S: X \rightarrow X$ are given mappings. Suppose that there exists a function $\psi \in \Psi$ such that

$$
\max \{p(T x, S y), p(S x, T y)\} \leq \psi(p(x, y))
$$

for all $x, y \in X$. Then $T$ and $S$ have a unique common fixed point.

Corollary 3.4 Let $(X, \vartheta)$ be a S-complete Hausdorff uniform space such that $p$ is an E-distance on $X$ and $T: X \rightarrow X$ be a given mapping. Suppose that there exists a function $\psi \in \Psi$ such that

$$
\max \{p(T x, y), p(x, T y)\} \leq \psi(p(x, y))
$$

for all $x, y \in X$. Then $T$ has a unique fixed point.

\subsection{Cyclic contraction on uniform space}

Corollary 3.5 Let $(X, \vartheta)$ be a S-complete Hausdorff uniform space such that $p$ is an E-distance on $X$ and $A_{1}, A_{2}$ are nonempty closed subsets of $X$ with respect to the topological space $(X, \tau(\vartheta))$. Let $T: Y \rightarrow Y$ be a mapping, where $Y=\bigcup_{i=1}^{2} A_{i}$. Suppose that the following conditions hold:

(i) $T\left(A_{1}\right) \subseteq A_{2}$ and $T\left(A_{2}\right) \subseteq A_{1}$;

(ii) there exists a function $\psi \in \Psi$ such that

$$
p(T x, T y) \leq \psi(p(x, y)), \quad \text { for all }(x, y) \in A_{1} \times A_{2} .
$$

Then $T$ has a unique fixed point that belongs to $A_{1} \cap A_{2}$.

Proof Since $A_{1}$ and $A_{2}$ are closed subsets of $X,(Y, d)$ is an $S$-complete Hausdorff uniform space. Define the mapping $\alpha: Y \times Y \rightarrow[0, \infty)$ by

$$
\alpha(x, y)= \begin{cases}1 & \text { if }(x, y) \in\left(A_{1} \times A_{2}\right) \cup\left(A_{2} \times A_{1}\right) \\ 0 & \text { otherwise }\end{cases}
$$


From (ii) and the definition of $\alpha$, we can write

$$
\alpha(x, y) p(T x, T y) \leq \psi(p(x, y))
$$

for all $x, y \in Y$. Thus $T$ is an $\alpha-\psi$-contractive mapping.

Let $(x, y) \in Y \times Y$ such that $\alpha(x, y) \geq 1$. If $(x, y) \in A_{1} \times A_{2}$, from (i), $(T x, T y) \in A_{2} \times A_{1}$, which implies that $\alpha(T x, T y) \geq 1$. If $(x, y) \in A_{2} \times A_{1}$, from (i), $(T x, T y) \in A_{1} \times A_{2}$, which implies that $\alpha(T x, T y) \geq 1$. Thus in all cases, we have $\alpha(T x, T y) \geq 1$. This implies that $T$ is $\alpha$-admissible.

Also, from (i), for any $a \in A_{1}$, we have $(a, T a) \in A_{1} \times A_{2}$, which implies that $\alpha(a, T a) \geq 1$. Now, let $\left\{x_{n}\right\}$ be a sequence in $X$ such that $\alpha\left(x_{n}, x_{n+1}\right) \geq 1$ for all $n$ and $x_{n} \rightarrow x \in X$ as $n \rightarrow \infty$. This implies from the definition of $\alpha$ that

$$
\left(x_{n}, x_{n+1}\right) \in\left(A_{1} \times A_{2}\right) \cup\left(A_{2} \times A_{1}\right), \quad \text { for all } n .
$$

Since $\left(A_{1} \times A_{2}\right) \cup\left(A_{2} \times A_{1}\right)$ is a closed subset of $X$ with respect to the topological space $(X, \tau(\vartheta))$, we get

$$
(x, x) \in\left(A_{1} \times A_{2}\right) \cup\left(A_{2} \times A_{1}\right),
$$

which implies that $x \in A_{1} \cap A_{2}$. Thus we can easily get from the definition of $\alpha$ that $\alpha\left(x_{n}, x\right) \geq 1$ for all $n$.

Finally, let $x, y \in \operatorname{Fix}(T)$. From (i), this implies that $x, y \in A_{1} \cap A_{2}$. So, for any $z \in Y$, we have $\alpha(z, x) \geq 1$ and $\alpha(z, y) \geq 1$. Thus condition (H) is satisfied.

Now, all the hypotheses of Theorem 2.6 are satisfied, and we deduce that $T$ has a unique fixed point that belongs to $A_{1} \cap A_{2}$ (from (i)).

\section{Competing interests}

The authors declare that there is no conflict of interests regarding the publication of this article.

\section{Authors' contributions}

All authors have equally contributed in writing this article. All authors have read and approved the final manuscript.

\section{Author details}

${ }^{1}$ Department of Mathematics, School of Natural Sciences, National University of Sciences and Technology, H-12, Islamabad, Pakistan. ${ }^{2}$ Department of Mathematics, Quaid-i-Azam University, Islamabad, Pakistan. ${ }^{3}$ Department of Mathematics, Atilim University, Incek, Ankara 06836, Turkey. ${ }^{4}$ Nonlinear Analysis and Applied Mathematics Research Group (NAAM), King Abdulaziz University, Jeddah, Saudi Arabia.

\section{Acknowledgements}

The authors thank the anonymous referees for their remarkable comments, suggestions, and ideas that helped to improve this paper.

Received: 1 April 2014 Accepted: 13 June 2014 Published: 22 Jul 2014

\section{References}

1. Samet, B, Vetro, C, Vetro, P: Fixed point theorems for $\alpha$ - $\psi$-contractive type mappings. Nonlinear Anal. 75, 2154-2165 (2012)

2. Karapınar, E, Samet, B: Generalized $\alpha$ - $\psi$-contractive type mappings and related fixed point theorems with applications. Abstr. Appl. Anal. 2012, Article ID 793486 (2012)

3. Asl, JH, Rezapour, S, Shahzad, N: On fixed points of $\alpha$ - $\psi$-contractive multifunctions. Fixed Point Theory Appl. 2012 , 212 (2012). doi:10.1186/1687-1812-2012-212

4. Mohammadi, B, Rezapour, S, Shahzad, N: Some results on fixed points of $\alpha-\psi$-Ciric generalized multifunctions. Fixed Point Theory Appl. 2013, 24 (2013). doi:10.1186/1687-1812-2013-24 
5. Ali, MU, Kamran, T: On $\left(\alpha^{*}, \psi\right)$-contractive multi-valued mappings. Fixed Point Theory Appl. 2013, 137 (2013). doi:10.1186/1687-1812-2013-137

6. Amiri, P, Rezapour, S, Shahzad, N: Fixed points of generalized $\alpha$ - $\psi$-contractions. Rev. R. Acad. Cienc. Exactas Fís. Nat., Ser. A Mat. (2013). doi:10.1007/s13398-013-0123-9

7. Minak, G, Altun, I: Some new generalizations of Mizoguchi-Takahashi type fixed point theorem. J. Inequal. Appl. 2013, 493 (2013). doi:10.1186/1029-242X-2013-493

8. Ali, MU, Kamran, T, Sintunavarat, W, Katchang, P: Mizoguchi-Takahashi's fixed point theorem with $\alpha, \eta$ functions Abstr. Appl. Anal. 2013, Article ID 418798 (2013)

9. Abdeljawad, T: Meir-Keeler $\alpha$-contractive fixed and common fixed point theorems. Fixed Point Theory Appl. 2013,19 (2013). doi:10.1186/1687-1812-2013-19

10. Karapınar, E, Kumam, P, Salimi, P: On $\alpha$ - $\psi$-Meir-Keeler contractive mappings. Fixed Point Theory Appl. 2013, 94 (2013). doi:10.1186/1687-1812-2013-94

11. Chen, CM, Karapınar, E: Fixed point results for the alpha-Meir-Keeler contractions on partial Hausdorff metric spaces. J. Inequal. Appl. 2013, 410 (2013). doi:10.1186/1029-242X-2013-410

12. Salimi, $\mathrm{P}$, Latif, A, Hussain, N: Modified $\alpha$ - $\psi$-contractive mappings with applications. Fixed Point Theory Appl. 2013 151 (2013). doi:10.1186/1687-1812-2013-151

13. Hussain, N, Salimi, P, Latif, A: Fixed point results for single and set-valued $\alpha-\eta-\psi$-contractive mappings. Fixed Point Theory Appl. 2013, 212 (2013). doi:10.1186/1687-1812-2013-212

14. Mohammadi, B, Rezapour, S: On modified $\alpha-\varphi$-contractions. J. Adv. Math. Stud. 6, 162-166 (2013)

15. Berzig, M, Karapınar, E: Note on 'Modified $\alpha-\psi$-contractive mappings with application'. Thai J. Math. 12 (2014)

16. Ali, MU, Kamran, T, Karapınar, E: $(\alpha, \psi, \xi)$-Contractive multi-valued mappings. Fixed Point Theory Appl. 2014, 7 (2014). doi:10.1186/1687-1812-2014-7

17. Ali, MU, Kamran, T, Karapınar, E: A new approach to $(\alpha, \psi)$-contractive nonself multivalued mappings. J. Inequal. Appl. 2014, 71 (2014) doi:10,1186/1029-242X-2014-71

18. Ali, MU, Kamran, T, Kiran, Q: Fixed point theorem for $(\alpha, \psi, \phi)$-contractive mappings on spaces with two metrics. J. Adv. Math. Stud. 7, 8-11 (2014)

19. Ali, MU, Kiran, Q, Shahzad, N: Fixed point theorems for multi-valued mappings involving $\alpha$-function. Abstr. Appl. Anal. 2014, Article ID 409467 (2014)

20. Kirk, WA, Srinivasan, PS, Veeramani, P: Fixed points for mappings satisfying cyclical weak contractive conditions. Fixed Point Theory 4, 79-89 (2003)

21. Karapınar, E: Fixed point theory for cyclic weak $\phi$-contraction. Appl. Math. Lett. 24, 822-825 (2011)

22. Karapınar, E, Sadarangani, K: Fixed point theory for cyclic $(\phi-\psi)$-contractions. Fixed Point Theory Appl. 2011, 69 (2011). doi:10.1186/1687-1812-2011-69

23. Pacurar, M, Rus, IA: Fixed point theory for cyclic $\varphi$-contractions. Nonlinear Anal. 72, 1181-1187 (2010)

24. Rus, IA: Cyclic representations and fixed points. Ann. 'Tiberiu Popoviciu' Sem. Funct. Equ. Approx. Convexity 3, 171-178 (2005)

25. Pacurar, M, Rus, IA: Fixed point theory for cyclic $\varphi$-contractions. Nonlinear Anal. 72, 1181-1187 (2010)

26. Hussain, N, Karapınar, E, Sedghi, S, Shobkolaei, N, Firouzain, S: Cyclic ( $\phi$ )-contractions in uniform spaces and related fixed point results. Abstr. Appl. Anal. 2014, Article ID 976859 (2014)

27. Bourbaki, N: Elements de mathematique. Fasc. II. Livre III: Topologie generale. Chapitre 1: Structures topologiques Chapitre 2: Structures uniformes, 4th edn. Actualités Scientifiques et Industrielles, vol. 1142. Hermann, Paris (1965)

28. Zeidler, E: Nonlinear Functional Analysis and Its Applications, vol. 1. Springer, New York (1986)

29. Aamri, M, El Moutawakil, D: Common fixed point theorems for E-contractive or E-expansive maps in uniform spaces. Acta Math. Acad. Paedagog. Nyházi. 20(1), 83-91 (2004)

30. Aamri, M, El Moutawakil, D: Weak compatibility and common fixed point theorems for A-contractive and E-expansive maps in uniform spaces. Serdica Math. J. 31, 75-86 (2005)

31. Aamri, M, Bennani, S, El Moutawakil, D: Fixed points and variational principle in uniform spaces. Sib. Electron. Math. Rep. 3, 137-142 (2006)

32. Agarwal, RP, O'Regan, D, Papageorgiou, NS: Common fixed point theory for multi-valued contractive maps of Reich type in uniform spaces. Appl. Anal. 83, 37-47 (2004)

10.1186/1687-1812-2014-150

Cite this article as: Ali et al.: Fixed point of $\alpha-\psi$-contractive type mappings in uniform spaces. Fixed Point Theory and Applications 2014, 2014:150 